\title{
AVANCES EN EL MEJORAMIENTO GENÉTICO DEL FRIJOL EN MÉXICO POR TOLERANCIA A TEMPERATURA ALTA Y A SEQUÍA
}

\author{
ADVANCES IN MÉXICO ON BEAN BREEDING FOR TOLERANCE TO HIGH TEMPERATURE \\ AND DROUGHT
}

\author{
Edwin J. Barrios Gómez ${ }^{1 \star}$, Cándido López Castañeda ${ }^{2}$, Josué Kohashi Shibata ${ }^{2}$, Jorge A. Acosta Gallegos ${ }^{3}$, \\ Salvador Miranda Colín ${ }^{1}$ y Netzahualcóyotl Mayek Pérez ${ }^{4}$
}

${ }^{1}$ Campo Experimental Zacatepec, Instituto Nacional de Investigaciones Forestales, Agrícolas y Pecuarias (INIFAP). Carr. 0.5 Zacatepec-Galeana. 62780 , Zacatepec,
Morelos, México. Tel. 73434 30230. Fax. 7343433820 . ${ }^{2}$ Postgrado en Recursos Genéticos y Productividad-Genética y ${ }^{2}$ Postgrado en Botánica, Colegio de
Postgraduados-Campus Montecillo. Km. 36.5 Carretera México-Texcoco. 56230, Montecillo, Texcoco, Estado de México. ${ }^{3}$ Campo Experimental Bajío, INIFAP.
Apdo. Postal 112. 38000, Celaya, Guanajuato, México. ${ }^{4}$ Centro de Biotecnología Genómica, Instituto Politécnico Nacional. 88710, Reynosa, Tamaulipas, México.

*Autor para correspondencia (barrios.edwin@inifap.gob.mx.)

\section{RESUMEN}

En el Altiplano Mexicano el frijol (Phaseolus vulgaris L.) tipo 'Flor de Mayo' es importante por su alta demanda para consumo humano. El objetivo del presente trabajo fue estudiar la respuesta a sequía y a temperaturas altas, así como el avance en el mejoramiento genético, en un grupo de cultivares de frijol tipo 'Flor de Mayo'. Se establecieron tres experimentos (primavera-verano de 2007): el primero en riego (Celaya, Guanajuato), el segundo y tercero en riego y secano respectivamente (Montecillo, Texcoco, Estado de México). Se incluyeron ocho variedades del tipo 'Flor de Mayo' (FM) y la variedad criolla 'Michoacán 128', todas de hábito indeterminado tipo III. Las variedades modernas mostraron mejores rendimientos de semilla y en sus componentes, así como en biomasa aérea final, y evidenciaron una considerable ganancia debida al mejoramiento genético, así como un avance en tolerancia a sequía sobre todo en el peso de 100 semillas. En cuanto al índice de susceptibilidad a sequía (ISS) para rendimiento de semilla (RS), biomasa aérea final $(\mathrm{BF})$, número de vainas $(\mathrm{NV})$, número de semillas (NS) y peso de 100 de semillas (P100S), se encontró que las variedades 'FM Sol' y 'FM M38' tuvieron valores menores a uno para RS, BF, NS y NV, y significativamente menores a las demás variedades $(P \leq 0.05)$, por lo que clasifican como resistentes a sequía. La variedad 'FM M38' mostró además el RS más alto en promedio de los tres experimentos. El diferencial de temperatura (T) del dosel del cultivo $\left(\mathrm{TDC}^{\circ} \mathrm{C}=\mathrm{T}\right.$ del aire - T del dosel del cultivo) fue mayor para 'FM Bajío, 'FM M38', 'FM Anita' y 'FM Noura', en promedio de los tres experimentos; los valores más bajos de TDC se observaron en las variedades 'FM 2000' y 'Michoacán 128', que tuvieron valores negativos (TDC por arriba de la $T$ del aire). La TDC se relacionó positiva y significativamente $(\mathrm{P} \leq 0.05)$ con RS, BF, NV y NS, donde los valores más altos correspondieron a las variedades con mejor rendimiento. El RS del frijol podría mejorarse mediante selección de genotipos con TDC mayor e ISS menor, en condiciones de estrés hídrico y temperaturas altas.

Palabras clave: Phaseolus vulgaris, sequía, temperatura alta, diferencial térmico del dosel, rendimiento de semilla.

\section{SUMARY}

Dry bean (Phaseolus vulgaris L.) of the 'Flor de Mayo' type is an important staple crop in the Mexican highlands because of its high demand for human consumption. The objective of this work was to study a group of 'Flor de Mayo' dry bean cultivars regarding their response to drought, high temperatures and breeding advances. Three experiments were established during 2007: Spring-Summer cycle at Celaya, Guanajuato under irrigated conditions, and at Montecillo, Texcoco, State of México under both rainfed and irrigated conditions. Eight 'Flor de Mayo' (FM) cultivars, along with 'Michoacán 128' dry bean landrace were evaluated, all of them of indeterminate type III growth habit. The modern varieties showed better seed yields and better yield components, as well as final aerial biomass, thus evidencing a considerable advance due to genetic improvement, which was associated to better tolerance to drought mainly in the weight of 100 seeds. On the drought susceptibility index (S) calculated for seed yield (SY), final aerial biomass (FB), number of pods (NP) number of seeds (NS) and weight of 100 seeds (W100S), varieties 'FM Sol' and 'FM M38' were classified as drought resistant with an $\mathrm{S}<1.0$ for SY, FB, NS and $\mathrm{NP}$, and significantly lower $(\mathrm{P} \leq \mathbf{0 . 0 5})$ than the other cultivar. Variety 'FM M38' showed the highest SY through the three experiments. The temperature (T) depression of the canopy ( $\mathrm{TDC}^{\circ} \mathrm{C}=$ air $\mathrm{T}$ - canopy T) was higher for 'FM Bajío, 'FM M38', 'FM Anita' and 'FM Noura', through the three experiments, with the lowest TDC values observed in varieties 'FM 2000' and 'Michoacán 128', which showed negative values (canopy temperature above of the air T). TDC was positive and significantly related with SY, FB, NP and NS, with the highest values corresponding to varieties with the best performance. Bean seed yield might be improved by selecting genotypes with higher TDC and lower $S$ under water stress and high temperatures.

Index words: Phaseolus vulgaris, drought, high temperature, thermal differential canopy, seed yield.

\section{INTRODUCCIÓN}

El frijol (Phaseolus vulgaris L.) crece en temperaturas promedio que oscilan de 15 a $27^{\circ} \mathrm{C}$, con una óptima de 25 ${ }^{\circ}$ C. Según Masaya y White (1991), el crecimiento vegetal responde a un intervalo de temperaturas críticas en función de los requerimientos de calor necesarios para el desarrollo; 
estas temperaturas incluyen a la temperatura base, óptima y la máxima. Las temperaturas altas incrementan la producción de yemas y flores, pero también la abscisión de botones florales, flores y vainas (Monterroso y Wien, 1990). Cuando el estrés por calor incide en prefloración sus efectos posteriores son más severos, como caída de vainas pequeñas y reducción del número de semillas, pero sin afectar al peso de semilla, en comparación con la incidencia en postfloración (Agtunong et al., 1992).

Las temperaturas muy altas pueden causar esterilidad de flores o inhibir la floración además de abatir la fijación de nitrógeno, porque afectan la actividad de Rhizobium y el abastecimiento de carbohidratos hacia los nódulos (Hungria y Antonio, 1993). La planta de frijol puede soportar temperaturas extremas entre 5 y $40{ }^{\circ} \mathrm{C}$ (Masaya y White, 1991), pero su tolerancia a las temperaturas altas es reducida, y la mayoría de variedades comerciales están adaptadas a temperaturas moderadas propias de regiones de elevación media a alta o de siembras de otoño-invierno. En soya (Glycine max L.) sometida a condiciones de riego y sequía, Harris et al. (1984) encontraron que la diferencia entre la temperatura del dosel del cultivo y la temperatura del aire varió de -2.6 en riego a $-0.2{ }^{\circ} \mathrm{C}$ en sequía, en promedio de todos los genotipos, y que su correlación con el rendimiento en el primer año fue negativa $(r=-0.69$, $\mathrm{P} \leq 0.01$ ), mientras que en el segundo año no se detectó asociación posiblemente porque hubo condiciones más favorables de precipitación.

El estrés por sequía es el factor abiótico que afecta en mayor grado la producción mundial de cultivos y en consecuencia la de alimentos. En México la sequía se debe principalmente a la escasa y errática distribución de la precipitación, que causan pérdidas que van desde 60 a $100 \%$ (Acosta et al., 2000). La tolerancia a sequía en especies vegetales es un proceso complejo que involucra diferencias morfológicas y anatómicas que contribuyen a la adaptación de la planta a condiciones restringidas de humedad. La sequía a menudo se presenta acompañada de temperaturas altas en áreas de temporal o secano (White y Singh, 1991). Según Fischer y Maurer (1978), la comparación del rendimiento entre sequía y riego mediante el índice de susceptibilidad a sequía (ISS) permite separar los efectos de tolerancia a sequía de aquellos debidos al escape y a las diferencias en rendimiento potencial; en estos términos, una menor susceptibilidad a sequía equivale a una mayor tolerancia. Para algunas variedades mexicanas de frijol, Rosales-Serna et al. (2000), Acosta-Díaz et al. (2004) y López-Salinas et al. (2008) encontraron que el índice de susceptibilidad a sequía permitió identificar variedades tolerantes a sequía.

Por otro lado, el alto contenido y la estabilidad de la clorofila en la hoja de varios cultivos son características que han mostrado una amplia relación con una alta capacidad fotosintética. La medición del contenido relativo de la clorofila con el SPAD ${ }^{\circledR}$ (Soil Plant Analysis Development), se ha propuesto en estudios de sequía como criterio para la selección de genotipos (Fotovat et al., 2007). Además, el contenido total de clorofila puede ser rápidamente determinado con el SPAD® (Arunyanark et al., 2008), y sus valores presentan una correlación alta con los valores determinados mediante el método de extracción de clorofila a, b y total, así como con la eficiencia transpiratoria (Arunyanark et al., 2008; Fotovat et al., 2007) y con el área foliar especifica (Fotovat et al., 2007), en diferentes condiciones de humedad.

En este estudio se compararon cultivares de frijol del tipo 'Flor de Mayo' registrados en México desde la década de 1970. Este tipo de frijol tiene actualmente el segundo lugar en superficie sembrada y consumo en la Zona Centro de México, y alcanza precios elevados. Hasta la fecha se cuenta con ocho variedades del tipo 'Flor de Mayo', y aunque se han estudiado en muchas investigaciones aún no se ha determinado el avance del mejoramiento de éstas en su conjunto. Así, el objetivo del presente trabajo fue evaluar en campo la respuesta a sequía, a altas temperaturas y su avance en el mejoramiento, de un grupo de cultivares de frijol tipo 'Flor de Mayo', así como explorar el efecto de temperaturas altas y de sequía en la intensidad del color verde en los foliolos (medida con el SPAD ${ }^{\circledR}$ ) y en el área foliar específica.

\section{MATERIALES Y MÉTODOS}

\section{Sitios experimentales}

Se llevaron a cabo tres experimentos en el ciclo primaveraverano (PV) del 2007; uno en Celaya, Guanajuato, México (203' LN y $100^{\circ} 50^{\prime} \mathrm{LO}$ y $1765 \mathrm{msnm}$ ) y los otros dos en Montecillo, Texcoco, Estado de México, México (19²1' LN y $98^{\circ} 55^{\prime}$ LO y 2250 msnm).

\section{Material genético}

Se incluyeron ocho variedades de frijol tipo 'Flor de Mayo' (FM) listadas en el Cuadro 1, que se utilizan en siembras comerciales de riego y de secano con buena precipitación en la región templada subhúmeda (Acosta-Gallegos et al., 2000; Rosales-Serna et al., 2004a), más la variedad criolla 'Michoacán 128' de hábito indeterminado procedente del Estado de Michoacán, México.

\section{Diseño experimental}

Para los tres experimentos se utilizó un diseño de bloques completos al azar con cuatro repeticiones, en lo que la 
Cuadro 1. Variedades de frijol utilizadas en tres sitios experimentales, ubicados en Celaya, Gto. y Montecillo, Texcoco, Edo. de México.

\begin{tabular}{llcc}
\hline Variedad & & $\begin{array}{c}\text { Año de } \\
\text { liberación }\end{array}$ & $\begin{array}{c}\text { Días a } \\
\text { floración }\end{array}$ \\
\hline 'Flor de Mayo M38' & 'FM M38' & 1994 & $50-57$ \\
'Flor de Mayo Corregidora' & 'FM Corregidora' & 2000 & 56 \\
'Flor de Mayo Sol' & 'FM Sol' & 1996 & $45-51$ \\
'Flor de Mayo RMC' & 'FM RMC' & 1981 & $44-55$ \\
'Flor de Mayo Bajío' & 'FM Bajío' & 1989 & $45-47$ \\
'Flor de Mayo Noura' & 'FM Noura' & 2006 & $47-50$ \\
'Flor de Mayo Anita' & 'FM Anita' & 2002 & $44-62$ \\
'Flor de Mayo 2000' & 'FM 2000' & 2001 & $45-50$ \\
'Michoacán 128'+† & & 1974 ' & $47-53$ \\
\hline
\end{tabular}

${ }^{\dagger}$ Variedades proporcionadas por el INIFAP, Celaya, Guanajuato. ${ }^{\dagger \dagger}$ Material proveniente de Michoacán, proporcionado por el Dr. Salvador Miranda Colín, Colegio de Postgraduados, Montecillo. "Año de colecta.

unidad experimental consistió de dos surcos de $0.80 \mathrm{~m}$ de separación y $4 \mathrm{~m}$ de longitud. En Celaya se aplicó riego antes de la siembra (3 de abril de 2007) en el fondo del surco; $15 \mathrm{~d}$ después de la siembra (dds) se hizo un aclareo para ajustar la población a 150000 plantas ha-1. Por falta de lluvia en Celaya se aplicaron cuatro riegos complementarios o de auxilio (condición de riego): a los 20 dds, crecimiento vegetativo (etapa R3), 55 y 65 dds, correspondientes a plena y final de floración (etapas R4 y R5). Los experimentos en Montecillo se sembraron el 12 de abril de 2007; en la condición de riego se aplicó de acuerdo con las necesidades del cultivo, y en el de secano se aplicó solamente hasta los 24 dds (25 d antes del inicio de la floración); el desarrollo posterior de las plantas dependió únicamente de la lluvia.

\section{Variables registradas y análisis estadístico}

Se determinó la temperatura del cultivo $\left(\mathrm{Tc},{ }^{\circ} \mathrm{C}\right)$ desde una distancia de $0.4 \mathrm{~m}$ del dosel con un termómetro infrarrojo (Raytek Spectrum Technologies, Inc.); las mediciones (un total de 16) se hicieron al medio día, entre las etapas de inicio de floración e inicio del crecimiento de las vainas. Durante estas mediciones prevalecieron días completamente soleados y viento con velocidad $<5 \mathrm{~km}$ $\mathrm{h}^{-1}$ En la madurez fisiológica se cosechó la porción aérea completa de las plantas en $1.0 \mathrm{~m}$ lineal de surco $\left(0.8 \mathrm{~m}^{2}\right)$ en cada unidad experimental, para determinar el rendimiento de semilla y sus componentes [vainas normales $\mathrm{m}^{-2}$ (VN $\left.\mathrm{m}^{-2}\right)$, semillas normales $\mathrm{m}^{-2}\left(\mathrm{SN} \mathrm{m} \mathrm{m}^{-2}\right)$, semillas por vaina (S/ vaina), peso de 100 semillas (P100S, g)] y biomasa aérea final (BAF, $\mathrm{g} \mathrm{m}^{-2}$ ). También se determinó el contenido de clorofila (unidades SPAD) con el SPAD 502®) (Minolta Corp., Ramsey, NJ), en cuatro fechas y 10 repeticiones (una repetición fue el foliolo central de una hoja) por parcela, para los experimentos de Celaya y Montecillo en riego y secano, entre los 50 y 70 dds.

El área foliar especifica $\left(\mathrm{AFE}, \mathrm{mg} \mathrm{cm}^{-2}\right)$ se determinó en tres hojas compuestas tomadas al azar por parcela, en las que se midió área foliar $\left(\mathrm{AF}, \mathrm{cm}^{2}\right)$ con un medidor de área foliar LI-3100® (LI-COR); posteriormente las muestras se secaron para obtener su peso seco (PSF, $\mathrm{mg}$ ); las mediciones en los tres experimentos se hicieron entre los 50 y 70 dds. Con el área foliar y el peso seco de las hojas se calculó el AFE mediante la expresión: AFE = PSF $(\mathrm{mg}) / \operatorname{AF}\left(\mathrm{cm}^{2}\right)$.

El índice de susceptibilidad a la sequía (ISS) se calculó para el rendimiento de semilla, biomasa aérea final, número de vainas $\mathrm{m}^{-2}$, número de semillas $\mathrm{m}^{-2} \mathrm{y}$ peso de 100 semillas, en los dos ambientes contrastantes (riego y secano), de acuerdo con Fischer y Maurer (1978): ISS $=[1-(Y / Y p)$ / 1 - $(X / X p)]$; donde, $Y$ es el rendimiento de semilla, sus componentes o biomasa aérea final, en secano o sequía; Yp es el rendimiento de semilla en riego; $X$ es el promedio del rendimiento de semilla en secano; y $X p$ es el rendimiento de semilla en riego. En la ecuación, el término [1 - $(X / X p)]$ corresponde al índice de intensidad a la sequía.

Con los datos se hicieron análisis de varianza para todas las variables medidas, con el programa SAS (SAS Institute, 2000) con un diseño en bloques completos al azar $\left(Y_{i j}=\mu+\right.$ $\left.T_{i}+B_{j}+E_{i j}\right)$ para cada experimento, y en forma combinada como serie de experimentos $\left(Y_{i j k}=\mu+L_{i}+T_{i}+L T_{i j}+B_{(i) j}\right.$ $\left.+E_{i j k}\right)$, para determinar las diferencias entre experimentos $(L)$, variedades $(T)$, y la interacción variedad $\mathrm{x}$ experimento $(L x T)$. Se calculó la diferencia significativa honesta de Tukey (DSH, $\mathrm{P} \leq 0.05$ ) para la comparación de medias.

\section{Contenido hídrico del suelo}

Se determinaron las curvas de retención de humedad y con éstas los puntos de marchitamiento permanente (PMP) y de capacidad de campo (CC) del suelo, en el Laboratorio de Física de Suelos del Colegio de Postgraduados, en muestras tomadas de cada una de las parcelas del experimento, antes de efectuar las siembras correspondientes. Semanalmente se determinó el porcentaje de humedad del suelo $(\% \mathrm{H})$ en las profundidades de 0-20, 20-40, 40-60 cm, desde la emergencia de las plántulas hasta la madurez, mediante el método gravimétrico después de secar las muestras de suelo a $110^{\circ} \mathrm{C}$ durante $48 \mathrm{~h}$ en una estufa (GCA Corporation, modelo 17). Los porcentajes de humedad se calcularon con la ecuación: \% H = [(PH - PS) 100)/ PS]; donde, $\mathrm{PH}$ es el peso (g) del suelo húmedo, y PS es el peso del suelo seco (g).

\section{Datos meteorológicos}

Los promedios mensuales de temperaturas máxima y mínima del aire y de precipitación acumulada se obtuvieron de dos estaciones meteorológicas ubicadas a una distancia de $200 \mathrm{~m}$ de los sitios experimentales (Cuadro 2). 


\begin{tabular}{|c|c|c|c|c|c|c|c|}
\hline Localidad & Marzo & Abril & Mayo & Junio & Julio & Media & Total \\
\hline \multicolumn{8}{|c|}{ Celaya, Guanajuato, México } \\
\hline Temperatura máxima $\left({ }^{\circ} \mathrm{C}\right)$ & 26.8 & 28.4 & 30.2 & 28.6 & 26.4 & 28.1 & - \\
\hline Temperatura mínima $\left({ }^{\circ} \mathrm{C}\right)$ & 8.2 & 9.6 & 12.9 & 14.6 & 14.1 & 11.9 & - \\
\hline Precipitación pluvial (mm) & 0.8 & 5.4 & 14.4 & 157.8 & 184.8 & - & 363.2 \\
\hline \multicolumn{8}{|c|}{ Montecillo, Texcoco, Edo. México } \\
\hline Temperatura máxima $\left({ }^{\circ} \mathrm{C}\right)$ & 30.7 & 31.7 & 32.9 & 32.6 & 30.4 & 31.7 & - \\
\hline Temperatura mínima $\left({ }^{\circ} \mathrm{C}\right)$ & 0.5 & 4.0 & 6.2 & 7.8 & 7.9 & 5.3 & - \\
\hline Precipitación pluvial (mm) & 19.6 & 50.7 & 46.3 & 143.3 & 152.1 & - & 412.0 \\
\hline
\end{tabular}

\section{RESULTADOS Y DISCUSIÓN}

\section{Condiciones ambientales}

La temperatura (T) óptima para el crecimiento del frijol es de $25^{\circ} \mathrm{C}$ (Masaya y White, 1991), con una T base de 8 ${ }^{\circ} \mathrm{C}$ (Barrios-Gómez y López-Castañeda, 2009). En los sitios experimentales la $\mathrm{T}$ promedio máxima del aire fue de 31.7 ${ }^{\circ} \mathrm{C}$ en Montecillo, y de $28.1^{\circ} \mathrm{C}$ en Celaya (Cuadro 2), ambas mayores que la óptima para el frijol. Las temperaturas máximas durante el periodo de mayor deficiencia hídrica edáfica (etapa R5) indujeron un alto nivel de estrés en las plantas, similar a lo indicado White y Singh (1991) y Wang et al. (2006). La T mínima promedio para Montecillo fue de $5.3{ }^{\circ} \mathrm{C}$ (Cuadro 2), menor que la $\mathrm{T}$ base de frijol; sin embargo, las plántulas no mostraron daños foliares (bordes de hojas y ápices quemados, cese del crecimiento). Otros investigadores (Machado et al., 2006; Barrios-Gómez y López-Castañeda, 2009) también han reportado que el frijol no ha mostrado daños por frio a temperaturas entre 4 y $10^{\circ} \mathrm{C}$.

En Celaya la $\mathrm{T}$ mínima promedio $\left(11.9^{\circ} \mathrm{C}\right)$ estuvo por arriba de la $\mathrm{T}$ base, lo que favoreció un rápido desarrollo de la planta. La distribución irregular de la lluvia se reflejó en un bajo contenido hídrico edáfico en el experimento de secano en Montecillo, con $25 \%$ de humedad en el estrato de 0 a $20 \mathrm{~cm}$ (valor de $\mathrm{PMP}=28 \%$ ) y con $32 \%$ de humedad en el estrato de 20 a $40 \mathrm{~cm}$ (valor de PMP $=34 \%$ ). En Celaya la humedad del suelo estuvo muy próxima al PMP (20\% de humedad) en la profundidad de 0 a $20 \mathrm{~cm}$ (valor de PMP =18) durante la etapa de inicio de floración e inicio del crecimiento de vainas, por lo que se le aplicaron riegos de auxilio.

\section{Índice de susceptibilidad a sequía (ISS)}

Los valores de este índice calculados para rendimiento de semilla, biomasa aérea final, número de vainas, número de semillas normales y peso de 100 de semillas, tuvieron valores menores $(\mathrm{P} \leq 0.01)$ en los genotipos 'FM Sol',
'FM M38' y 'FM Corregidora' que las demás variedades, pero solamente en las dos primeras estos estuvieron por debajo de la unidad. Estas variedades se clasificaron como tolerantes a sequía porque presentaron menores ISS que los otros cultivares $(\mathrm{P} \leq 0.01)$ (Figura 1$)$. La variedad 'FM M38' fue la que mostró el rendimiento más alto de semilla en promedio de los tres experimentos, mientras que 'FM Sol' y 'FM Corregidora' tuvieron rendimientos bajos aunque sus valores de ISS fueron bajos (tolerantes a sequía). De manera similar, Rosales-Serna et al. (2000) y López Salinas et al. (2008) señalaron que si bien el ISS es un criterio aceptable para seleccionar genotipos que reduzcan menos su rendimiento en condiciones de estrés hídrico, no necesariamente éstos serán los de mayor rendimiento.

En relación al año de liberación de las variedades, si ISS mostró ciertas consistencias, ya que para rendimiento de semilla y biomasa aérea final las variedades modernas 'FM Sol', 'FM M38' y 'FM Corregidora' mostraron valores $<$ a 1.0, mientras que para peso de 100 semillas, además de éstas, 'FM Noura' y 'FM Anita' también mostraron valores de ISS $<$ a 1.0, lo que sugiere que también son tolerantes a sequía para estas características. Sin embargo, para número de vainas 'FM Corregidora' y 'FM Noura', y para número de semillas normales 'FM M38', fueron solamente las únicas variedades tolerantes.

\section{Diferencia de la temperatura del dosel}

El análisis estadístico mostró efectos significativos $(\mathrm{P} \leq$ 0.01 ) de los tratamientos en la diferencia de la temperatura del dosel (datos no mostrados), donde el de Montecillo en riego $\left(2.1^{\circ} \mathrm{C}\right)$ presentó los valores más altos, seguido por Celaya $\left(1.6^{\circ} \mathrm{C}\right)$ y por último el de Montecillo en secano $(-2.0$ $\left.{ }^{\circ} \mathrm{C}\right)$; el valor negativo indica que la temperatura de la planta estuvo por arriba de la temperatura del aire (Cuadro 3). Las variedades 'FM Bajío,' 'FM M38' y 'FM Noura' de Montecillo en secano fueron las que presentaron temperaturas menores que el aire, y cuando más se acercaron a $0{ }^{\circ} \mathrm{C}$, en cambio, las variedades 'Michoacán 128' y 'FM 2000' mostraron temperaturas superiores a las del aire (Cuadro 3). 


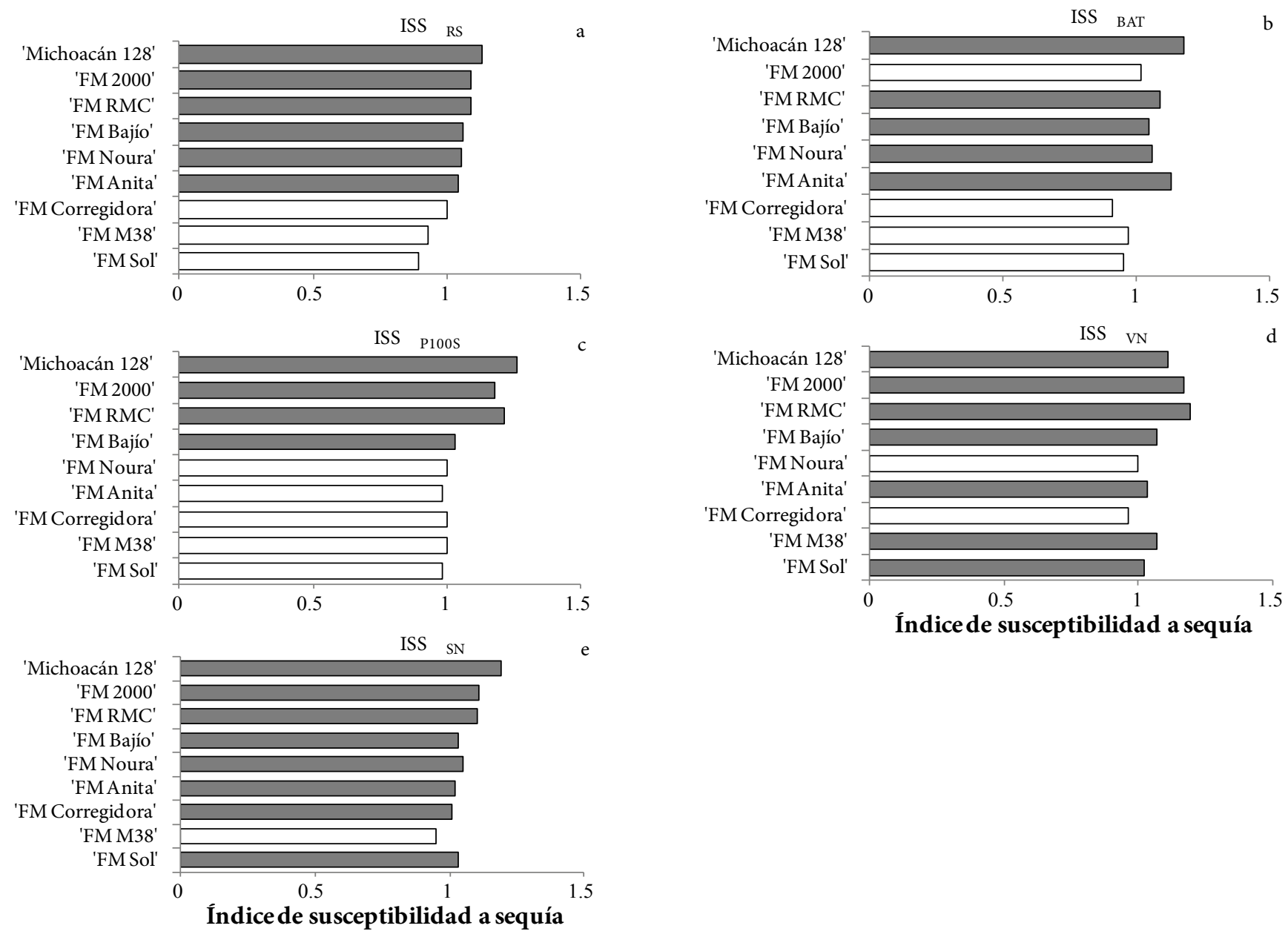

Figura 1. Índice de susceptibilidad a sequía (ISS) para las variables: rendimiento de semilla (a), biomasa aérea final (b), peso de 100 semillas (c), vainas normales $\mathrm{m}^{-2}(\mathrm{~d})$, y semillas normales $\mathrm{m}^{-2}(\mathrm{e}) .2007$. Barras rellenas, ISS $>1.0$; barras vacías ISS $<1.0$.

Cuadro 3. Promedios de diferenciales de la temperatura del dosel del cultivo y de rendimiento de semilla (RS, $\mathrm{g} \mathrm{m}^{-2}$ ) en tres sitios ubicados en Celaya, Gto. y Montecillo, Texcoco, Edo. de México, 2007.

\begin{tabular}{|c|c|c|c|c|c|}
\hline \multirow{3}{*}{ Variedades } & \multicolumn{4}{|c|}{ Diferencia de la temperatura del dosel $\left({ }^{\circ} \mathrm{C}\right)$} & \multirow{3}{*}{$\mathrm{RS}\left(\mathrm{g} \mathrm{m}^{-2}\right)$} \\
\hline & \multicolumn{2}{|c|}{ Montecillo } & \multirow{2}{*}{$\begin{array}{l}\text { Celaya } \\
\text { Secano }\end{array}$} & \multirow{2}{*}{ Promedio } & \\
\hline & Riego & Secano & & & \\
\hline 'FM Noura' & 2.7 & -1.5 & 2.1 & 1.1 & 317.4 \\
\hline 'FM Anita' & 2.1 & -1.9 & 3.2 & 1.1 & 301.0 \\
\hline 'FM M38' & 2.7 & -1.4 & 2.0 & 1.1 & 291.8 \\
\hline 'FM Sol' & 2.4 & -1.7 & 1.4 & 0.7 & 237.5 \\
\hline 'FM Bajío’ & 2.5 & -0.3 & 2.1 & 1.4 & 217.6 \\
\hline 'FM Corregidora' & 2.0 & -2.8 & 1.7 & 0.3 & 209.9 \\
\hline 'FM 2000’ & 0.8 & -3.0 & 0.3 & -0.7 & 209.5 \\
\hline 'FM RMC' & 2.1 & -2.4 & 1.3 & 0.3 & 179.1 \\
\hline 'Michoacán 128' & 1.7 & -2.6 & 0.6 & -0.1 & 142.6 \\
\hline DHS $(\mathrm{P} \leq 0.01)$ & 1.5 & 1.0 & 1.8 & 1.0 & 67.1 \\
\hline Media general & 2.1 & -2.0 & 1.6 & 0.6 & 234.0 \\
\hline
\end{tabular}


En promedio de variedades en los tres experimentos, 'FM Bajío,' 'FM M38', 'FM Anita' y 'FM Noura' tuvieron mayor diferencial de temperatura del dosel $(\mathrm{P} \leq 0.05)$ que las otras variedades; los valores más bajos $(\mathrm{P} \leq 0.01)$ se observaron en los cultivares 'FM 2000' y 'Michoacán 128', que incluso presentaron valores negativos. El contraste entre las condiciones favorables (Montecillo en riego y Celaya en riego) y la condición menos favorable (Montecillo en secano), puede deberse en gran medida a que en este ultimo hubo deficiencias hídricas edáficas ( $20 \%$ de humedad, por abajo del PMP que fue de $28 \%$, entre los 43 a 63 dds en el estrato de 0 a $20 \mathrm{~cm}$ ) así como incidencia de temperaturas más altas que la óptima $\left(25^{\circ} \mathrm{C}\right)$ durante las etapas de floración y llenado de semilla. En el ambiente de Montecillo en secano las plantas experimentaron un déficit de humedad severo durante la floración y la etapa de crecimiento de vainas, en las dos profundidades de suelo: entre 0 y $20 \mathrm{~cm}, 8 \%$ por abajo del PMP; entre 20 y $40 \mathrm{~cm}, 4$ $\%$ menos que el valor de PMP.
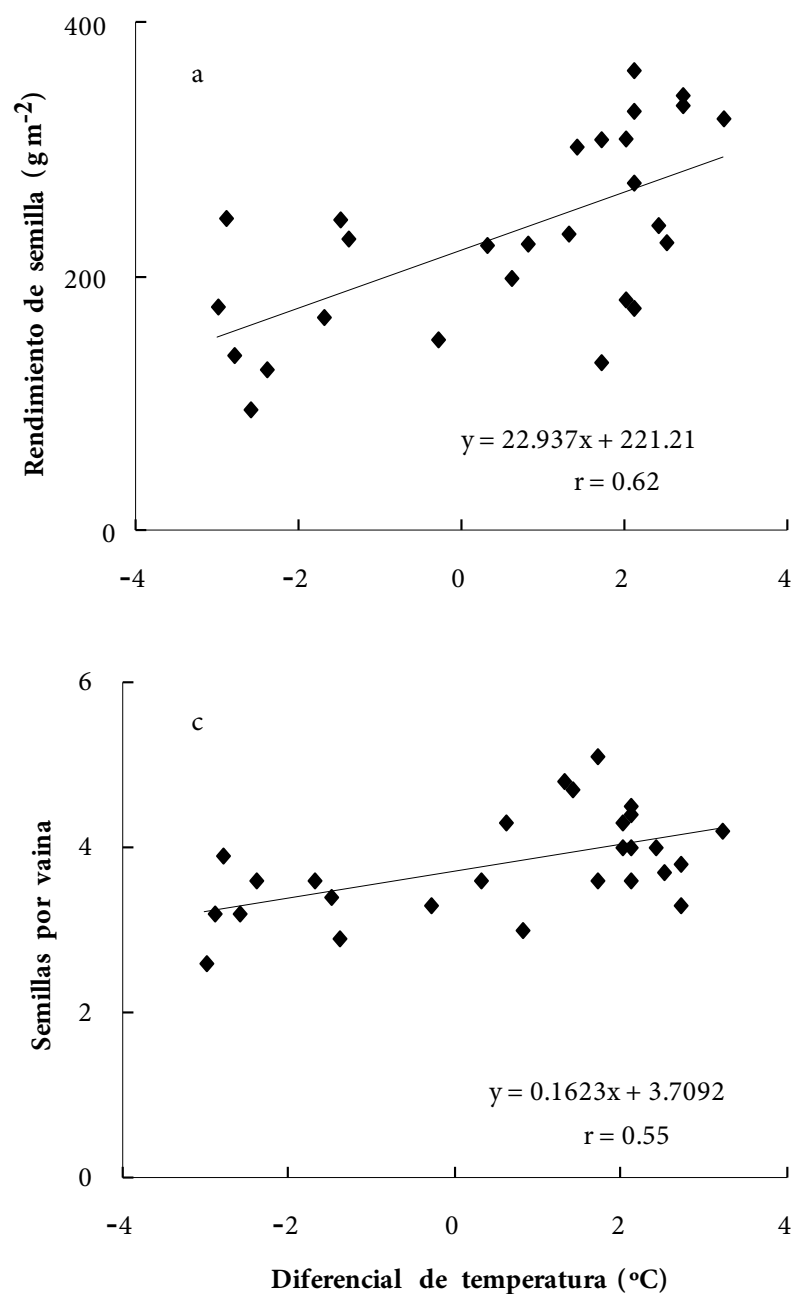

El diferencial de temperatura del dosel se relacionó linealmente $(\mathrm{P} \leq 0.01)$ con rendimiento de semilla (Figura 2a), biomasa aérea final (Figura $2 \mathrm{~b}$ ), semillas por vaina (Figura 2c), número de semillas (Figura 2d), y $(\mathrm{P} \leq 0.05)$ con número de vainas (gráfica no mostrada), con coeficientes de correlación desde 0.55 a 0.62 . Estas correlaciones indican que a mayor diferencial térmico, las variedades de frijol tendrán mayores valores de rendimiento de semilla, biomasa aérea final, vainas normales $\mathrm{m}^{-2}$ y semillas normales $\mathrm{m}^{-2}$. En trigo (Triticum aestivum L.) también se ha reportado una relación positiva y significativa del diferencial térmico con el rendimiento, la biomasa, granos $\mathrm{m}^{-2}$, espigas $\mathrm{m}^{-2}$, granos/espiga y días a floración (Reynolds et al., 2001). En el presente estudio, las variedades que tuvieron el mayor diferencial térmico tal vez sea debido a que mantuvieron sus estomas más abiertos y así propiciaron mayor intercambio gaseoso, mayor conductancia estomática y capacidad fotosintética, en comparación con las variedades de menor
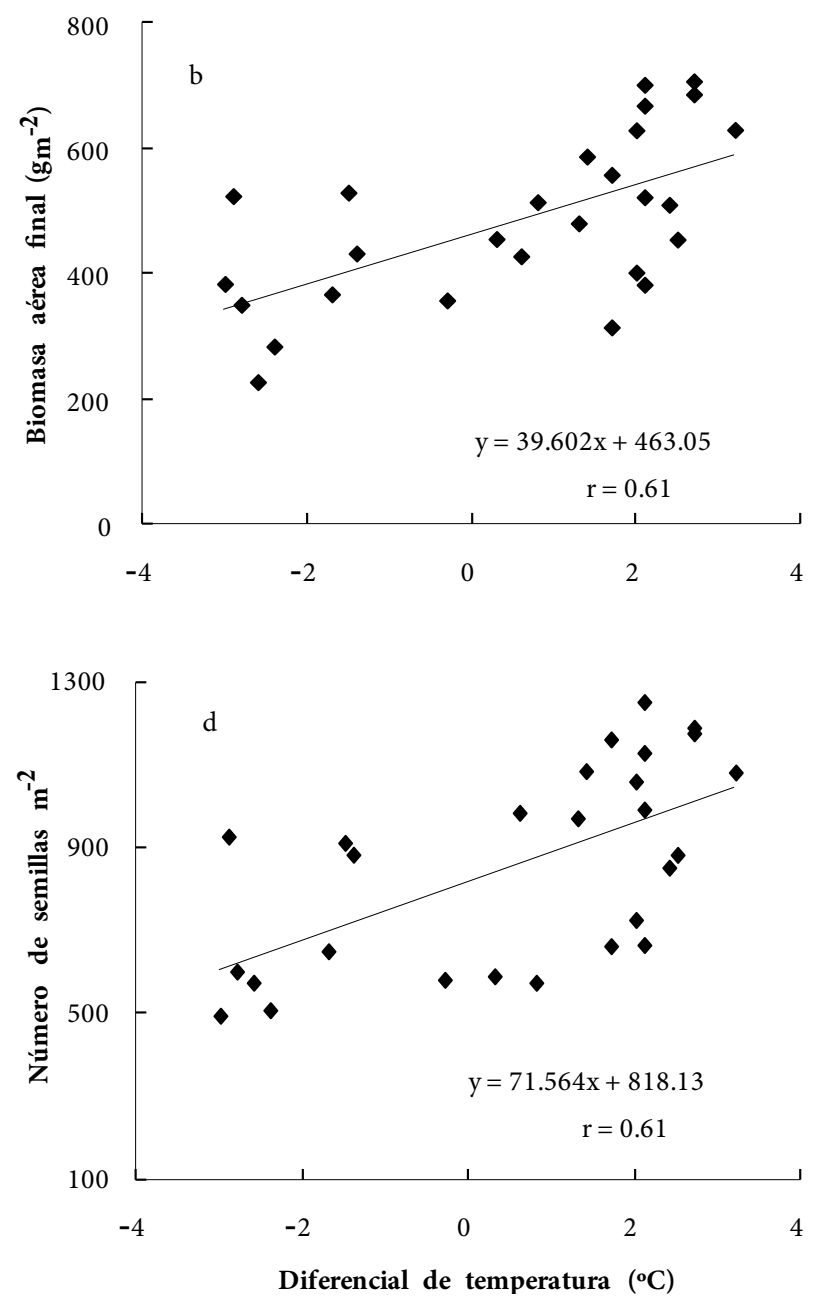

Figura 2. Relación entre el diferencial de temperatura del dosel y las variables rendimiento de semilla (a), biomasa aérea final (b), semillas por vaina (c) y número de semillas $\mathbf{m}^{-2}$ (d), en Montecillo en riego, Montecillo en secano y Celaya en riego, 2007 (n=27). 
diferencial, como propusieron Reynolds et al. (2001). En frijol se ha indicado que las altas temperaturas $\left(>30^{\circ} \mathrm{C}\right.$ ) reducen el número de semillas, número de vainas, peso promedio de semillas y semillas por vaina, con pérdidas promedio de $83,63,47$, y $73 \%$, respectivamente (Rainey y Griffiths, 2005). Las temperaturas altas también reducen la viabilidad del polen y el crecimiento del tubo polínico (Weaver et al., 1985), causan aborto de flores y vainas jóvenes, y reducen el número de semillas por vaina, el tamaño de semilla y el rendimiento de semilla (Shonnard y Gepts, 1994). Estos efectos pudieron haber ocurrido en este trabajo de investigación.

\section{Relación entre el año de liberación de las variedades y el rendimiento y sus componentes}

El análisis de esta relación permitió detectar que las variedades más recientes ('FM Noura' y 'FM M38') poseen mayores $(\mathrm{P} \leq 0.01)$ tasas de ganancia en rendimiento de semilla (Figura 3a), biomasa aérea final (Figura 3b) $\mathrm{y}$ en el peso de 100 semillas (Figura 3c). Este análisis también muestra la ganancia de rendimiento atribuible al mejoramiento genético, que colateralmente se asocia con un aumento en la biomasa aérea final y en el tamaño de semilla. Podría ser conveniente entonces que en la selección de futuras variedades se considere la inclusión de la biomasa aérea final y del peso de 100 semillas como criterios de selección, además de variables de calidad como el contenido y calidad de proteína, oxidación del grano, entre otras. La contribución de la biomasa aérea final y el número de vainas normales $\mathrm{m}^{-2}$ al rendimiento de semilla también es importante en ambientes con déficit hídrico. En Urbana, Illinois, EE. UU., se determinó que los cultivares modernos de soya 'Clark 63' y 'Williams 82' en sequía produjeron 9 $\%$ más rendimiento de semilla que los cultivares antiguos 'Manchu' y 'Dunfield', debido a que tenían una mayor biomasa aérea final y número de vainas $\mathrm{m}^{-2}$ (Frederick et al., 1991). Al estudiar las variedades de frijol liberadas en 54 años, del tipo Pinto perteneciente a la raza Durango (Oeste de EE. UU.), Singh et al. (2007) encontraron una ganancia del rendimiento de $35 \%$.

El ISS para peso de 100 semillas mostró correlación significativa $(\mathrm{P} \leq 0.05, \mathrm{r}=0.63)$ con el año de liberación de las variedades. En las variables rendimiento de semilla, número de semillas $\mathrm{m}^{-2}$, número de vainas ${ }^{-2} \mathrm{y}$ biomasa aérea final el ISS no mostró una relación significativa con el año de liberación aunque como las pendientes tendieron a ser negativas es posible que las variedades de reciente liberación sean un poco más tolerantes a sequía que las variedades antiguas.

\section{Contenido relativo total de clorofila (SPAD) y área foliar específica (AFE)}

Los análisis de varianza para AFE y valores del SPAD mostraron diferencias $(\mathrm{P} \leq 0.01)$ entre sitios experimentales $\mathrm{y}$ variedades, pero la interacción sitios $\mathrm{x}$ variedades no fue significativa, por lo que aquí se presentan en forma conjunta. Entre sitios, Montecillo en secano presentó los valores mayores de AFE con una media de $5.6 \mathrm{mg} \mathrm{cm}^{-2}$, seguido por Celaya con 4.4, y por último Montecillo en riego con $3.9 \mathrm{mg} \mathrm{cm}^{-2}$. Entre variedades, 'FM Corregidora' mostró el valor mayor $(\mathrm{P} \leq 0.01)$ de AFE, junto con 'FM Bajío, 'FM M38' y 'FM Noura'; las variedades con menor AFE fueron 'Michoacán 128' y 'FM RMC'. Aunque algunas variedades de alto rendimiento de semilla tuvieron valores altos de AFE, no hubo correlación significativa entre estas dos características.

Entre sitios los valores más altos de clorofila correspondieron a Montecillo en secano con un valor

Cuadro 4. Área foliar específica y contenido de clorofila (unidades SPAD) por sitio experimental y en promedio de los tres experimentos efectuados en Celaya, Gto. y Montecillo. Edo. de México, 2007.

\begin{tabular}{|c|c|c|c|c|c|c|c|c|}
\hline \multirow[b]{2}{*}{ Variedades } & \multicolumn{4}{|c|}{ Area foliar específica $\left(\mathrm{mg} \mathrm{cm}^{-2}\right)$} & \multicolumn{4}{|c|}{ Contenido de clorofila (SPAD) } \\
\hline & $\begin{array}{l}\text { Celaya } \\
\text { Riego }\end{array}$ & $\begin{array}{l}\text { Montecillo } \\
\text { Riego }\end{array}$ & $\begin{array}{l}\text { Montecillo } \\
\text { Secano }\end{array}$ & Promedio & $\begin{array}{l}\text { Celaya } \\
\text { Riego }\end{array}$ & $\begin{array}{l}\text { Montecillo } \\
\text { Riego }\end{array}$ & $\begin{array}{c}\text { Montecillo } \\
\text { Secano }\end{array}$ & Promedio \\
\hline 'FM Sol' & 4.7 & 3.8 & 5.8 & 4.8 & 46.5 & 43.6 & 46.5 & 45.5 \\
\hline 'FM Corregidora' & 4.7 & 4.2 & 6.2 & 5.0 & 44.8 & 43.7 & 46.2 & 44.9 \\
\hline 'FM Bajío’ & 5.2 & 4.1 & 6.1 & 5.1 & 45.4 & 43.6 & 45.1 & 44.7 \\
\hline 'FM Noura' & 4.2 & 4.1 & 5.6 & 4.6 & 43.3 & 42.4 & 44.2 & 43.3 \\
\hline 'FM Anita & 4.6 & 4.0 & 6.0 & 4.9 & 43.9 & 42.0 & 43.4 & 43.1 \\
\hline ‘FM M38’ & 4.5 & 3.9 & 5.9 & 4.7 & 41.9 & 42.1 & 43.6 & 42.5 \\
\hline 'FM 2000’ & 4.2 & 3.7 & 4.7 & 4.2 & 41.3 & 39.8 & 42.4 & 41.2 \\
\hline 'FM RMC' & 4.0 & 3.8 & 5.8 & 4.5 & 39.6 & 38.6 & 42.9 & 40.4 \\
\hline ‘Michoacán 128’ & 3.8 & 3.7 & 4.7 & 4.1 & 39.8 & 37.5 & 41.6 & 39.6 \\
\hline $\mathrm{DHS}(\mathrm{P} \leq 0.05)^{\dagger}$ & 1.2 & 0.9 & 1.8 & 1.3 & 2.2 & 3.3 & 4.1 & 0.8 \\
\hline Promedio & 4.4 & 3.9 & 5.6 & 4.7 & 42.9 & 41.5 & 44.0 & 42.8 \\
\hline DHS $(\mathrm{P} \leq 0.05)^{\dagger \dagger}$ & & 0.6 & & - & & 0.8 & & - \\
\hline
\end{tabular}

${ }^{\dagger}$ Para comparar genotipos dentro de cada experimento; ${ }^{\dagger \dagger}$ Para comparar promedios entre experimentos. 

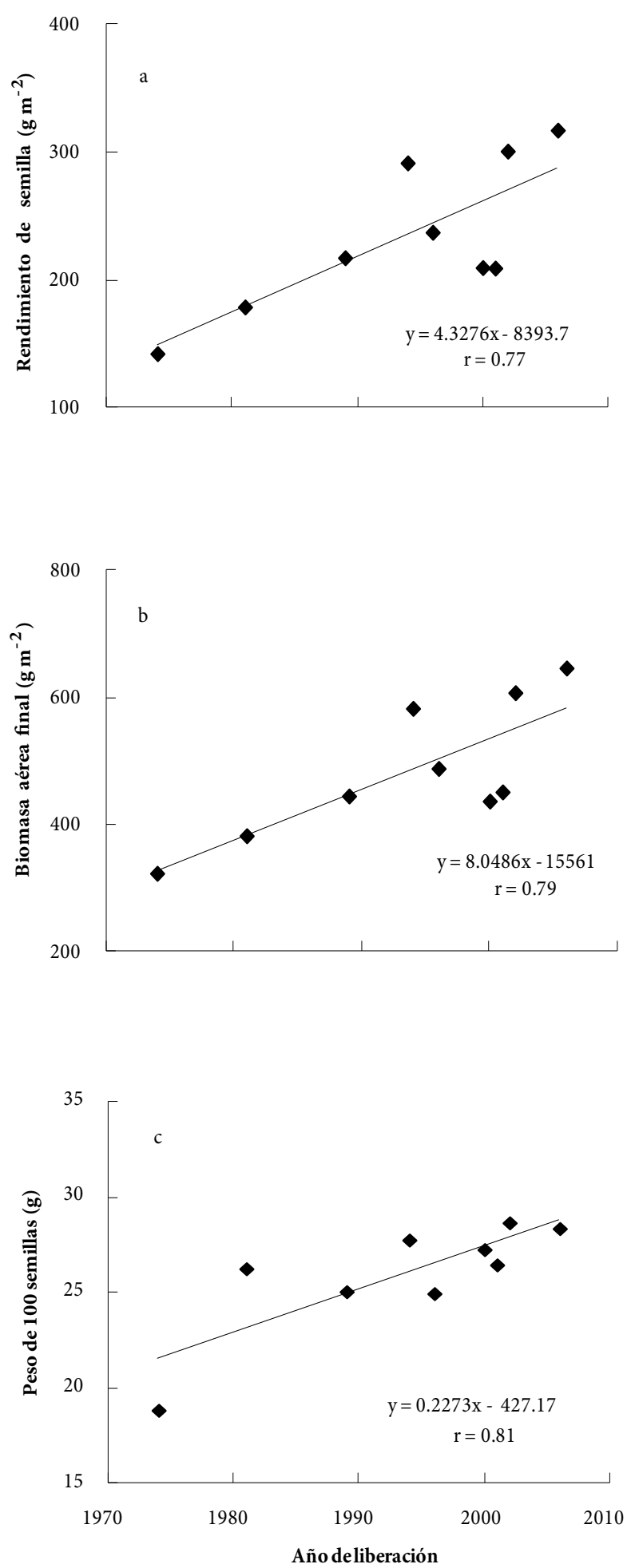

Figura 3. Relación entre año de liberación de nueve variedades con su rendimiento de semilla (a), biomasa aérea final (b) y peso de 100 semillas (c), 2007. de 44.0 seguido de Celaya en riego (42.9), y el valor más bajo fue el de Montecillo en riego con 41.5. Se detectaron diferencias en contenido de clorofila en función del nivel de humedad en el suelo, con los valores más altos en el sitio que tuvo el mayor déficit hídrico, resultado que no concuerda con lo encontrado en frijol por Rosales-Serna $e t$ al. (2004b), quienes no detectaron diferencias considerables entre condiciones de humedad, aunque sí entre variedades.

En promedio de variedades los valores más altos ( $\mathrm{P}$ $\leq 0.01$ ) se presentaron en 'FM Sol', 'FM Bajío' y 'FM Corregidora', y los más bajos en 'FM Anita', 'FM Noura' y 'FM M38'. Entre sitios, el contenido de clorofila fluctúo de 40 a 46 unidades SPAD. Estos valores encontrados en frijol son menos heterogéneos que los reportados por Giunta et al. (2002) en cebada (Hordeum vulgare L.) con oscilaciones de 33 a 40, de 49 a 54 en trigo y de 45 a 50 en triticale (x Triticosecale Wittmack); según estos autores, la utilización del SPAD puede ayudar en la discriminación preliminar de variedades con alto y bajo contenido de clorofila. El SPAD también se ha propuesto como criterio de selección de genotipos bajo condiciones de sequía en cacahuate (Arachis hypogaea L.) (Fotovat et al., 2007) y en trigo (Arunyanark et al., 2008), pero su utilidad en la selección de variedades de frijol por resistencia a sequía es dudosa (Rosales-Serna et al., 2004b).

En el estudio que aquí se presenta se encontró una alta correlación positiva $(\mathrm{r}=0.86 \mathrm{P} \leq 0.01$, ) entre los valores del SPAD y el AFE, en concordancia con lo encontrado en cacahuate (Nageswara et al., 2001). Algunas variedades que tuvieron altos rendimientos de semilla también tuvieron valores altos de AFE y SPAD, pero esto no fue generalizado.

\section{CONCLUSIONES}

Las variedades de frijol 'FM Bajío, 'FM M38', 'FM Anita' y 'FM Noura' exhibieron mejor tolerancia a temperaturas altas, en contraste con 'FM 2000' y 'Michoacán 128' que tuvieron la respuesta menos favorable.

Las variedades modernas mostraron mejores rendimientos de semilla y sus componentes, así como de biomasa aérea final, lo que evidencia un considerable avance en el mejoramiento genético. Este mejoramiento también a producido ganancias colaterales en tolerancia a sequía, sobre todo en el peso de 100 semillas. El índice de susceptibilidad a sequía permitió identificar a las variedades 'FM Sol', 'FM M38' y 'FM Corregidora' como las más tolerantes a sequía. De estas tres solamente 'FM M38' mostró rendimiento alto en promedio de los tres sitios experimentales.

El índice de susceptibilidad a sequía podría utilizarse en la selección de nuevas variedades, que junto con las 
características agronómicas de interés y una menor interacción genotipo $\mathrm{x}$ ambiente, pueden constituir criterios de selección para el mejoramiento del rendimiento del frijol en áreas de secano. El contenido relativo de clorofila y el área foliar específica son características también afectadas por la falta de humedad en el suelo, pero que en este estudio no tuvieron relación con el rendimiento ni con la tolerancia a sequía.

\section{BIBLIOGRAFÍA}

Acosta-Díaz E, C Trejo-López, L Ruiz-Posadas, S Padilla-Ramírez, J A Acosta-Gallegos (2004) Adaptación del frijol a sequía en la etapa reproductiva. Terra 22:49-58.

Acosta-Gallegos J A, R Rosales-Serna, R Navarrete-Maya, E LópezSalinas (2000) Desarrollo de variedades mejoradas de frijol para condiciones de riego y temporal en México. Agric. Téc. Méx. 26:79-98.

Agtunong T P, R Redden, M A Mengee-Nang, C Searle, S Fukai (1992) Genotypic variation in response to high temperature at flowering in common bean (Phaseolus vulgaris L.). Aust. J. Exp. Agric. 32:1135-1140

Arunyanark A, S Jogloy, C Akkasaeng, N Vorasoot, T Kesmala, R C Nageswara Rao, G C Wright, A Patanothai (2008) Chlorophyll stability is an indicator of drought tolerance in peanut. J. Agron. Crop Sci. 194:113-125.

Barrios-Gómez E J, C López-Castañeda (2009) Temperatura base y tasa de extensión foliar. Agrociencia 43:29-35.

Fischer R A, R Maurer (1978) Drought resistance in spring wheat cultivars. I. Grain yield responses. Aust. J. Agric. Res. 29:897-912.

Frederick J R, J T Wooley, J D Hesketh, D B Peters (1991) Seed yield and agronomic traits of old and modern soybean cultivars under irrigation and soil water-deficit. Field Crops Res. 27:71-82.

Fotovat R, M Valizadeh, M Toorchi (2007) Association between wateruse efficiency components and total chlorophyll content (SPAD) in wheat (Triticum aestivum L.) under well-watered and drought stress conditions. J. Food Agric. Environ. 5:225-227.

Giunta F, R Motzo, M Deidda (2002) SPAD readings and associated leaf traits in durum wheat, barley and triticale cultivars. Euphytica 125:197-205.

Harris D S, W T Schapaugh Jr, E T Kanemasu (1984) Genetic diversity in soybeans for leaf canopy temperature and the association of leaf canopy temperature and yield. Crop Sci. 24:839-842.

Hungria M, A Antonio Franco (1993) Effects of high temperature on nodulation and nitrogen fixation by Phaseolus vulgaris L. Plant and Soil 149:95-102.

López-Salinas E, O H Tosquy Valle, F J Ugalde Acosta, J A Acosta Gallegos (2008) Rendimiento y tolerancia a sequía de genotipos de frijol negro en el Estado de Veracruz. Rev. Fitotec. Mex. 31:3539.

Masaya P, J W White (1991) Adaptation to photoperiod and temperature. In: Common Beans: Research for Crop Improvement. A V Schoonhoven, O Voysest (eds). C. A. B. Intl. U. K. and CIAT, Cali, Colombia. pp:445-500

Machado N N B, M Regina P, A B Gatti, V J Mendes C (2006) Temperature effects on seed germination in races of common beans (Phaseolus vulgaris L.). Acta Scin. Agron. 28:155-164.

Monterroso V A, H C Wien (1990) Flower and pod abscission due to heat stress in beans. J. Amer. Soc. Hort. Sci. 115:631-634.

Nageswara R R C, H S Talwar, G C Wright (2001) Rapid assessment of specific leaf area and leaf nitrogen in peanut (Arachis hypogaea $\mathrm{L}$.) using a chlorophyll meter. J. Agron. Crop Sci. 186:175-182.

Rainey K M, P D Griffiths (2005) Differential response of common bean genotypes to high temperature. J. Amer. Soc. Hort. Sci. 130:18-23.

Reynolds M P, S Nagarajan, M A Razzaque, O A A Ageeb (2001) Heat tolerance. In: Application of Physiology in Wheat Breeding. Reynolds M P, J I Ortiz-Monasterio, A McNab (eds). CIMMYT. México, D. F. pp:124-135.

Rosales-Serna R, P Ramírez-Vallejo, J A Acosta-Gallegos, F CastilloGonzález, J D Kelly (2000) Rendimiento de grano y tolerancia a sequía del frijol común en condiciones de campo. Agrociencia 34: 153-165.

Rosales S R, J A Acosta G, J Muruaga S, J Hernández M, G Esquivel, P Pérez (2004a) Variedades mejoradas de frijol del Instituto Nacional de Investigaciones Forestales, Agrícolas y Pecuarias. Libro Técnico No. 6. SAGARPA, INIFAP, CIRCE, Campo Experimental Valle de México. Chapingo. México. México. 148 p.

Rosales-Serna R, J Kohashi-Shibata, J A Acosta-Gallegos, C TrejoLópez, J Ortiz-Cereceres, J D Kelly (2004b) Biomass distribution, maturity acceleration and yield in drought-stressed bean cultivars. Field Crops Res. 85:203-211.

Singh S P, H Terán, M Lema, D M Webster, C A Strausbaugh, P N Miklas, H F Schwartz, M A Brick (2007) Seventy-five years of breeding dry bean of the western USA. Crop Sci. 47:981-989.

Shonnard G C, P Gepts (1994) Genetics of heat tolerance during reproductive development in common bean. Crop Sci. 34:11681175.

SAS Institute Statistical Analysis System (2000) Versión 8 para Windows Inc., Cary, NC, USA.

Wang J, Y T Gan, F Clarke, C L McDonald (2006) Response of chickpea yield to high temperature stress during reproductive development. Crop Sci. 46:2171-2178.

White J W, S P Singh (1991) Breeding for adaptation to drought. In: Common Beans: Research for Crop Improvement. A V Schoonhoven, O Voysest (eds). C. A. B. Intl. U. K. and CIAT, Cali, Colombia. pp:501-560.

Weaver M L, H Timm, D W Burke (1985) Pollen staining and high temperature-tolerance of bean. J. Amer. Soc. Hort. Sci. 110:797799. 